\title{
Optical observations of Comet 67P/Churyumov-Gerasimenko
}

\author{
M. Weiler ${ }^{1}$, H. Rauer ${ }^{1}$, and J. Helbert ${ }^{2}$ \\ 1 Institute of Planetary Research, DLR, Rutherfordstrasse 2, 12489 Berlin, Germany \\ 2 Optical Information Systems and Space Sensor Technologies, DLR, Rutherfordstrasse 2, 12489 Berlin, Germany
}

Received 5 August 2003 / Accepted 13 October 2003

\begin{abstract}
From longslit spectra obtained during the 1996 perihelion passage of Comet 67P/Churyumov-Gerasimenko, a $\mathrm{CN}$ production rate of $(1.35 \pm 0.35) \times 10^{25}$ molecules $\mathrm{s}^{-1}$ was determined and an upper limit for the $\mathrm{C}_{3}$ production rate of $1 \times 10^{25}$ molecules s ${ }^{-1}$ was derived. The dust color and dust activity were determined from the continuum light in the spectra. A neutral dust color was determined between $4070 \AA$ and $4600 \AA$. The CN production rate and the Af $\rho$ parameter, determined to $(428.2 \pm 59.8) \mathrm{cm}$, were similar to the values of the 1982 perihelion passage, indicating that the comet's activity did not change significantly in recent orbital passages. A first crude estimate of the dust production rate indicates a high dust to gas mass ratio.

$R$-filter images taken in March 2003 were used to study the dust coma morphology. Two jet structures were seen in the coma; these structures show no variations within the observing period from March 7 to May 30, 2003. The orientation of these stationary structures suggests an inclination of the rotation axis of the nucleus of approximately $40^{\circ}$ with respect to the orbital plane.
\end{abstract}

Key words. comets: general - comets: individual: 67P/Churyumov-Gerasimenko

\section{Introduction}

The Jupiter family comet 67P/Churyumov-Gerasimenko (hereafter $67 \mathrm{P} / \mathrm{C}-\mathrm{G})$ is the new target for the Rosetta space mission (ESA). Since the knowledge about the properties and the long-term behavior of this comet is poor, detailed studies of comet $67 \mathrm{P} / \mathrm{C}-\mathrm{G}$ are necessary.

From HST observations by Lamy et al. (2003), the effective radius of the nucleus of $67 \mathrm{P} / \mathrm{C}-\mathrm{G}$ was determined to $(1.98 \pm 0.02) \mathrm{km}$ and a rotation period of $(12.3 \pm 0.27) \mathrm{h}$ was derived. Comet $67 \mathrm{P} / \mathrm{C}-\mathrm{G}$ has an orbital period of approximately 6.6 years. Due to the geometrical orientation of Earth, Sun and 67P/C-G, the observing conditions were good only every second perihelion passage, otherwise the solar elongation of the comet during perihelion passage was too small. Production rates available in literature (e.g. Lowell Observatory Cometary Database (LOCD), described by A'Hearn et al. 1995; Cochran et al. 1992) for OH, CN, $\mathrm{C}_{3}$, $\mathrm{C}_{2}$ and $\mathrm{NH}$ are derived from observations during the 1982 apparition. Based on observations in 1982, comet 67P/C-G was found to be depleted in carbon-bearing molecules by Osip et al. (1992) and A'Hearn et al. (1995).

In this work, we present production rates of $\mathrm{CN}$ and an upper limit for the $\mathrm{C}_{3}$ production at the 1996 perihelion passage.

Send offprint requests to: $\mathrm{M}$. Weiler,

e-mail: michael.weiler@dlr.de

* Based on observations collected at the Observatoire de Haute-Provence (OHP), France, and Thüringer Landessternwarte Tautenburg (TLS), Germany.
Furthermore, broadband filter images obtained in spring 2003 reveal a dust anti-tail and coma structures.

\section{Observations and data reduction}

In February 1996, longslit spectra of comet 67P/C-G were obtained at the Observatoire de Haute-Provence (OHP), France. At the $1.93 \mathrm{~m}$ telescope at OHP the medium resolution longslit spectrograph CARELEC (Lemaitre et al. 1990) was used for the observations. The instrument was equipped with a $512 \times$ 512 pixel CCD, providing a slit length of 5.5 arcmin and a spatial scale of $1.1^{\prime \prime} /$ pixel. The slitwidth used was $2.1^{\prime \prime}$. The slit was aligned along the projected solar direction. The instrument set-up is summarized in Table 1. During the three nights of observations, different wavelengths ranges have been chosen to cover various emission bands in the optical spectrum of the comet. The wavelengths ranges and observational details are given in Table 2. Unfortunately, only on February 10/11, 1996 the sky conditions were photometric.

Standard data reduction routines were applied to the spectra including bias subtraction, flatfielding, wavelength calibration, sky background subtraction and extinction correction. In order to separate the gaseous emissions from the background of scattered sunlight on cometary dust particles, a solar analogue spectrum (Hyades VB 64) obtained with the same instrument was fitted to the cometary spectra by using a polynomial. The fitted solar analogue spectrum was subtracted from the cometary spectra to separate the gas emissions and the continuum of scattered sunlight. 
Table 1. Technical parameters of the observations using the instrument CARELEC at the $1.93 \mathrm{~m}$ telescope at OHP and the CCD camera at the $2 \mathrm{~m}$ telescope of TLS.

\begin{tabular}{lcccccc}
\hline \hline Instrument & Date & $\begin{array}{c}\text { Slit length } \\
{\left[{ }^{\prime}\right]}\end{array}$ & $\begin{array}{c}\text { Slit width } \\
{\left[{ }^{\prime \prime}\right]}\end{array}$ & $\begin{array}{c}\text { Spatial scale } \\
{\left[{ }^{\prime \prime} / \text { pixel }\right]}\end{array}$ & $\begin{array}{c}\text { Wavelengths scale } \\
{[\AA \text { 年/pixel }]}\end{array}$ & $\begin{array}{c}\text { FOV } \\
{\left[{ }^{\prime}\right]}\end{array}$ \\
\hline CARELEC & February, 1996 & 5.5 & 2.1 & 1.1 & 1.8 & - \\
TLS-CCD 1 & March 06/07, 2003 & - & - & 1.5 & - & 52.6 and 28.9* \\
TLS-CCD 2 & other & - & - & 1.1 & - & 38.2 and $21.0^{*}$ \\
\hline
\end{tabular}

${ }^{*} \mathrm{CCD}$ area reduced to save readout time in some exposures.

Table 2. Spectroscopic observations of comet $67 \mathrm{P} / \mathrm{C}-\mathrm{G}$ from OHP. All observations were performed at the $1.93 \mathrm{~m}$ telescope using the CARELEC spectrograph. $r_{\mathrm{h}}$ and $\Delta$ denote the heliocentric and geocentric distance, respectively, and $\beta$ denotes the phase angle. $N$ is the number of spectra obtained in one night, $T$ the total exposure time during the night and $\Delta \lambda$ is the wavelengths range of the spectra.

\begin{tabular}{lcccccc}
\hline \hline Date & $r_{\mathrm{h}}[\mathrm{AU}]$ & $\Delta[\mathrm{AU}]$ & $\beta\left[^{\circ}\right]$ & $N$ & $T[\mathrm{~min}]$ & $\Delta \lambda$ \\
\hline $09 / 10.02 .1996^{*}$ & 1.33 & 1.18 & 45.9 & 2 & 20 & $5817 \AA-6731 \AA$ \\
$10 / 11.02 .1996$ & 1.33 & 1.18 & 45.7 & 3 & 50 & $3751 \AA-4666 \AA$ \\
$11 / 12.02 .1996^{*}$ & 1.34 & 1.19 & 45.6 & 4 & 50 & $6034 \AA-6944 \AA$ \\
\hline
\end{tabular}

${ }^{*}$ Night not photometric.

Table 3. Broadband filter observations of comet $67 \mathrm{P} / \mathrm{C}-\mathrm{G}$ with the $2 \mathrm{~m}$-telescope at TLS. All observations were performed with the $2 \mathrm{~m}$ telescope/CCD camera. Observation dates and time intervals of the observations are given. $r_{\mathrm{h}}$ and $\Delta$ denote the heliocentric and geocentric distance, $\beta$ the phase angle, $N$ the number of images and $T$ the exposure time of each frame. None of the observations were obtained under photometric conditions.

\begin{tabular}{lccccccc}
\hline \hline Date & Time $[\mathrm{UT}]$ & $r_{\mathrm{h}}[\mathrm{AU}]$ & $\Delta[\mathrm{AU}]$ & $\beta\left[{ }^{\circ}\right]$ & $N$ & Filter & $T[\mathrm{~min}]$ \\
\hline 07.03 .2003 & $01: 52-02: 47$ & 2.47 & 1.49 & 4.3 & 14 & $B+R$ & 2 \\
27.03 .2003 & $20: 36-21: 41$ & 2.62 & 1.69 & 10.0 & 19 & $R$ & 2 \\
28.03 .2003 & $00: 26-00: 53$ & 2.62 & 1.70 & 10.1 & 10 & $R$ & 2 \\
28.03 .2003 & $20: 17-21: 03$ & 2.63 & 1.71 & 10.4 & 15 & $R$ & 2 \\
31.03 .2003 & $21: 41-22: 27$ & 2.65 & 1.75 & 11.4 & 15 & $R$ & 2 \\
30.05 .2003 & $21: 00-22: 26$ & 3.06 & 2.82 & 19.3 & 17 & $V+R$ & 2 \\
\hline
\end{tabular}

In March and May, 2003, $B, V$ and $R$-filter images of comet $67 \mathrm{P} / \mathrm{C}-\mathrm{G}$ were obtained using the $2 \mathrm{~m}$-telescope of the Thüringer Landessternwarte Tautenburg (TLS), Germany. The observations are listed in Table 3. For the technical parameters of the instrument used see Table 1.

The images were bias subtracted, flatfield corrected and the sky background was subtracted. Since all observations were performed through thin clouds, no absolute flux calibration is possible. It was not possible to track the comet with the telescope, therefore a number of short time exposures were taken and co-added for image analysis. During the exposure time of each image, the comet's movement is less than the spatial resolution of the CCD. All images taken in one of the six time intervals given in Table 3 were co-added after shifting the images to compensate the comet's movement.

The broadband filter observations were affected by some technical problems. The night of March 6/7, 2003 was dedicated to the testing of a new CCD-chip (CCD 1 in Table 1). This chip produced some interference patterns varying with time. These structures are only weak (less than $2 \%$ of the maximum comet intensity in a 2 min exposure) and large compared to the extension of the coma of comet $67 \mathrm{P} / \mathrm{C}-\mathrm{G}$. Therefore, they do not disturb the analysis of the coma for spatial structures in the observations of this night.

In the nights of March 6/7, 27/28 and 28/29 problems with the electronic equipment lead to a number of pixels in every exposure having the value zero. When several exposures of comet $67 \mathrm{P} / \mathrm{C}-\mathrm{G}$ were added, the resulting image contained so many of these pixels that the analysis of the coma became difficult. Interpolating the values of the disturbed pixels from the surrounding area of the same image is not suitable in the inner coma of the comet since the intensity gradient is large, making it difficult to obtain a good fit. Therefore, the positions of the disturbed pixels were detected by applying a cut-off level in each image. The pixel values were replaced by values derived from another (in most cases the next following) image. 
In order to take the changing weather conditions into account, the values from the image used for replacing the disturbed pixels were multiplied by a correction factor. This factor was determined from the fluxes of a number of stars, integrated in both the image to be corrected and the image used for the replacement. Due to the shifting of the images on sub-pixel level to compensate the comet's movement, the pixels neighbouring a disturbed pixel could be affected as well. Therefore, all pixels of an image surrounding a detected "bad" pixel were also replaced.

Images obtained on May 30, 2003 could not be used for coma analysis. The comet faded significantly from the previous observations in March, so no sufficient signal to noise ratio could be archived. The strong loss in brightness was in part caused by the increase of the geocentric distance by approximately a factor of two and increase of the phase angle from $4.3^{\circ}$ to $19.3^{\circ}$ between beginning of March and end of May. Since we have no absolute measure of the comet's gas or dust activity we cannot quantify how much of the brightness decrease is due to decreasing cometary activity.

During our observation period Earth crossed the orbital plane of comet $67 \mathrm{P} / \mathrm{C}-\mathrm{G}$, moving from $4.3^{\circ}$ south of the plane on March 7 to $0.8^{\circ}$ north of the plane on May 30, measured from the comet's nucleus. This means, the dust tail of comet $67 / \mathrm{C}-\mathrm{G}$ is seen nearly edge-on in our observations. At the same time comet $67 \mathrm{P} / \mathrm{C}-\mathrm{G}$ was at a high elongation $\left(169^{\circ}\right.$ on March 7 , decreasing to $94^{\circ}$ at the end of May). This leads to unusual position angles of the solar direction with respect to the dust tail direction. The projected Sun direction moved in direction of the extended tail structure during our observations while the position of the tail changed only a few degrees with respect to the equatorial coordinates. When the Earth was in the orbital plane of the comet on May 10/11, the tail structure should have pointed directly along the projected Sun direction. The neck-line structure is therefore strictly speaking an antitail. Because of its strong variations in our observations, the projected Sun direction is not a suitable reference direction in this work.

\section{Gas production rates}

The longslit spectra of February 10/11, 1996 were used to determine the production rate of $\mathrm{CN}$ from the observed $\left(\mathrm{B}^{2} \Sigma^{+}-\right.$ $\left.\mathrm{X}^{2} \Sigma^{+}\right)$emission band at $3880 \AA$ using a Haser model. Figure 1 shows a spectrum of comet $67 \mathrm{P} / \mathrm{C}-\mathrm{G}$ after continuum subtraction. To increase the signal to noise ratio the spectrum is averaged from $3766 \mathrm{~km}$ to $7531 \mathrm{~km}$ projected nucleocentric distance on the tailward side of the nucleus for display. The $\mathrm{C}_{3}$ emission $\left(\tilde{\mathrm{A}} \Pi_{\mathrm{u}}-\tilde{\mathrm{X}}^{1} \Sigma_{\mathrm{g}}^{+}\right.$system at $4050 \AA$ ) could not be clearly detected. Therefore, only an upper limit for the $\mathrm{C}_{3}$ production rate was derived.

The Haser scale lengths for CN from Fink et al. (1991) are used and a gas velocity of $1 \mathrm{~km} \mathrm{~s}^{-1}$ is assumed. The g-factor is derived from Schleicher (1983). For $\mathrm{C}_{3}$, the scale lengths and the g-factor from A'Hearn et al. (1995) are applied.

For the determination of the production rates, all three spectra obtained on February 10/11, 1996 were added, leading to an effective exposure time of $50 \mathrm{~min}$.

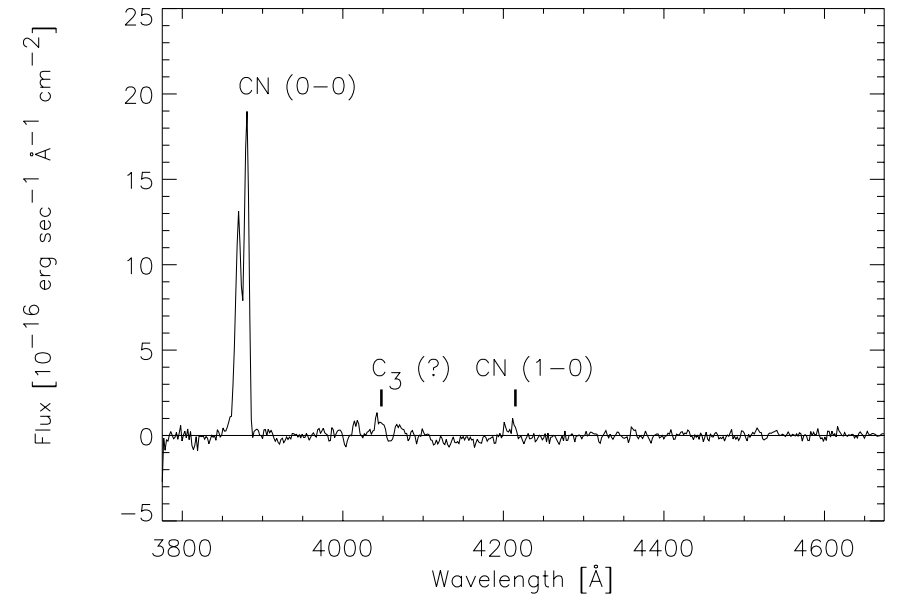

Fig. 1. Spectrum of comet 67P/C-G, obtained on February 10, 1996 and averaged between $3766 \mathrm{~km}$ and $7531 \mathrm{~km}$ projected nucleocentric distance on the tailward side of the nucleus for better display. The continuum is subtracted.

A CN production rate of $(1.35 \pm 0.35) \times 10^{25}$ molecules $\mathrm{s}^{-1}$ was computed from the spectra, averaged over the sunward and tailward side of the nucleus. Using scale lengths from other authors (A'Hearn et al. 1995; Cochran 1985; Rauer et al. 2003) leads to less good fits to the radial intensity profiles, but the effect on the production rates is less than $20 \%$.

An upper limit for the $\mathrm{C}_{3}$ production rate of $1 \times$ $10^{25}$ molecules s $^{-1}$ was determined.

For comparison, the $\mathrm{CN}$ production rates from the 1982 perihelion passage of comet 67P/C-G are shown in Fig. 2 (given by LOCD and Cochran et al. 1992) together with the value presented in this work. A perihelion asymmetry in the $\mathrm{CN}$ activity can be seen in the data from the 1982 perihelion passage. Comparing the $\mathrm{CN}$ production rates from 1982 with our value from 1996, no significant change in activity occured.

\section{Dust color and activity}

The color of a cometary coma is defined by Jewitt \& Meech (1986). The dust color was determined on the wavelengths interval [4070 ̊, $4600 \AA$ ] from our longslit spectra. We compared directly to the solar spectrum instead of using the analogue star which can show slight deviations from solar color. A solar catalogue spectrum (Kurucz et al. 1984) was brought to the same resolution as the comet spectra and the flux calibrated cometary spectra were divided by the solar spectrum to obtain the reflectivity. This was done for a mean spectrum of the cometary coma, obtained by adding the innermost 12 columns on both sides of the nucleus, corresponding to $\pm 1.1 \times 10^{4} \mathrm{~km}$ projected nucleocentric distance. Within the wavelengths interval a weak $\mathrm{CN}$ emission and a remnant of a night sky line were present. Nevertheless, a good linear fit to the reflectivity was possible on this wavelengths range. By using the flux calibrated spectra instead of the fitted continuum spectra, we avoided additional errors due to continuum fitting.

A mean color of $(-0.7 \pm 5.1) \% / 10^{3} \AA$ was determined on the interval [4070 $\AA, 4600 \AA$ ] . 


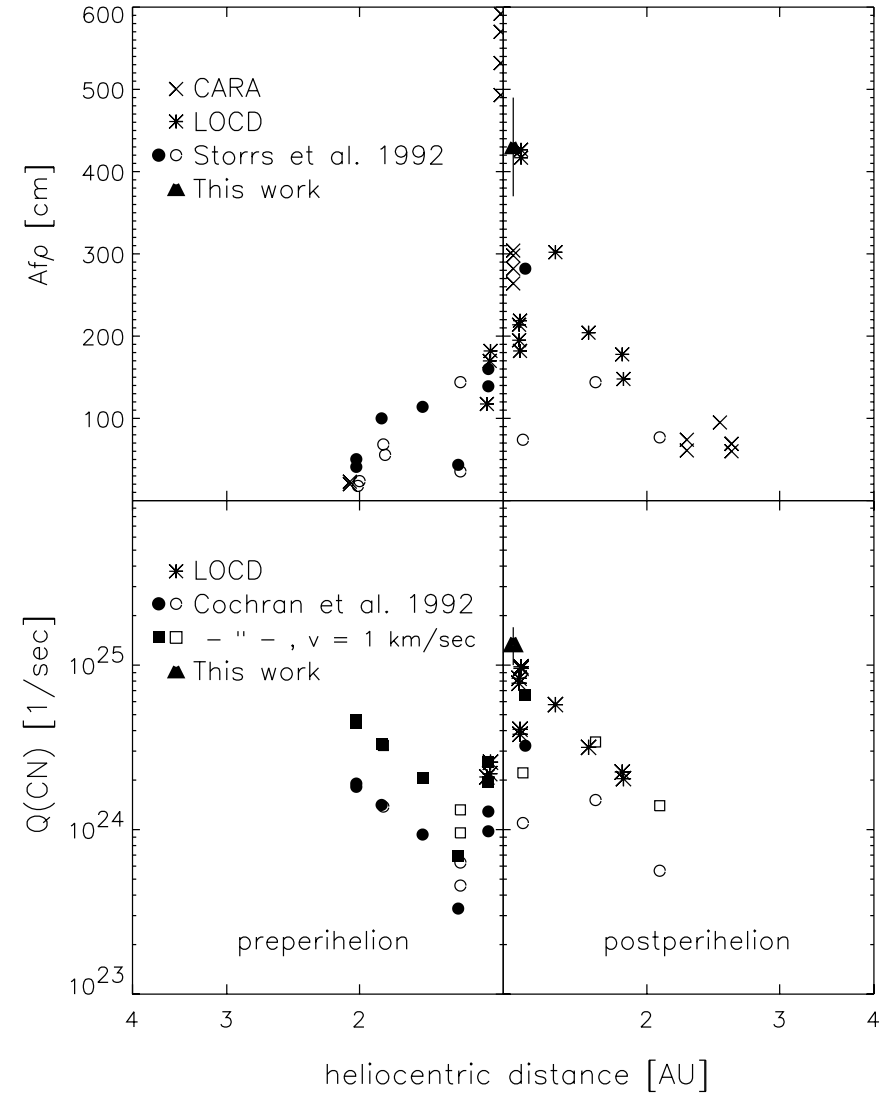

Fig. 2. Upper panel: Af $\rho$ values for comet $67 \mathrm{P} / \mathrm{C}-\mathrm{G}$. The data from LOCD and Storrs et al. (1992) are from the 1982 perihelion passage. CARA marks values from the Cometary Archive for Amateur Astronomer of the Italian Comet Section (Sezione Comete UIA). Note that all preperihelion values from CARA are from the 1996 apparition and all postperihelion values from this reference are from the 2002 apparition. The triangle gives $\operatorname{Af} \rho$ from this work for the 1996 perihelion passage. The Af $\rho$ parameters are determined at a wavelength of $5240 \AA$ by Storrs et al. (1992) from the flux through a continuum filter with transmission between $4770 \AA$ and $4830 \AA$ in the LOCD and at $4160 \AA$ in this work. CARA values are for various wavelengths, all in the red wavelengths range. Lower panel: $\mathrm{CN}$ production rates from the 1982 perihelion passage (LOCD, Cochran et al. (1992)) and for the 1996 perihelion passage (triangle). Open symbols in the data by Storrs et al. (1992) and Cochran et al. (1992) mark data from nights without photometric conditions. Squares give the $\mathrm{CN}$ production rates from Cochran et al. (1992), corrected to a constant gas velocity of $1 \mathrm{~km} \mathrm{~s}^{-1}$, as used in the LOCD data.

The Af $\rho$ parameter, as defined by A'Hearn et al. (1984), was determined from the longslit spectra at a wavelength of $4160 \AA$. Near this wavelength no strong gaseous emissions are present. To derive $\operatorname{Af} \rho$, it was assumed that the cometary coma was rotationally symmetric. The spatial intensity profile was extracted from the longslit spectra. Then, the hypothetical flux through a diaphragm with a projected radius, $\rho$, of $5 \times 10^{4} \mathrm{~km}$ was computed and used for the determination of $\operatorname{Af} \rho$. This was done for the sunward and tailward side of the longslit spectra independently to estimate the influence of the coma asymmetry.
A mean Af $\rho$ value of $428.2 \mathrm{~cm}$ was determined with a deviation of $\pm 59.8 \mathrm{~cm}$ on the sunward and tailward side of the slit due to coma asymmetries.

The Af $\rho$ parameter derived from longslit spectra is high but of the same order of magnitude as $\operatorname{Af} \rho$ determined at the 1982 perihelion passage by LOCD and Storrs et al. (1992) at similar heliocentric distances (see Fig. 2). Unfortunately, due to the lack of images it can not be ruled out that $\operatorname{Af} \rho$ from the longslit spectra is influenced by a strongly asymmetric coma.

The dust production rate can be determined from $\operatorname{Af} \rho$ if a number of assumptions are made. We used the model described by Weiler et al. (2003) to determine the dust mass production rate and the maximum grain size for particles that can be lifted from the nucleus surface. This is done by solving the Eulerequations for the gas flow, treating the dust grains as test particles in the flow to compute the velocities of dust grains in the inner coma. We used the $\mathrm{OH}$ production rates given by the LOCD as an estimate of the water production rates, assumed an active surface fraction of $10 \%$ as an upper limit and not varying along the orbit, a radius of $2 \mathrm{~km}$ (Lamy et al. 2003) and a density of $1 \mathrm{~g} \mathrm{~cm}^{-3}$ for the nucleus, and a dust density function according to Newburn \& Spinrad (1985). With these parameters one obtains a maximum radius of particles that can be lifted from the surface by the gas flow against gravity of $9.5 \mathrm{~cm}$ at the maximum of activity on December 14, 1982, at 1.36 AU postperihelion. This maximum radius decreases to $0.8 \mathrm{~cm}$ at $1.5 \mathrm{AU}$ postperihelion in March, 1983.

In order to determine the dust production rate, we assume the "standard" values for the parameters needed. A dust size distribution according to Newburn \& Spinrad (1985) was applied, assuming a peak in the size distribution at $0.4 \mu \mathrm{m}$ and a decrease with an exponent of -3.5 . A Bond albedo of 0.2 and a phase function according to Divine (1981) are used. We used the $\operatorname{Af} \rho$ values from the LOCD for which $\mathrm{OH}$ production rates are measured at the same time. Other species, like $\mathrm{CO}$, are neglected here. A peak dust production rate of $2.08 \times 10^{3} \mathrm{~kg} \mathrm{~s}^{-1}$ in mid December 1982 is determined with those standard parameters at $1.36 \mathrm{AU}$ postperihelion. The dust production decreases to $95 \mathrm{~kg} \mathrm{~s}^{-1}$ at $1.85 \mathrm{AU}$ postperihelion in 1983 . These values are higher than dust production rates published previously. The maximum dust production rates are $220 \mathrm{~kg} \mathrm{~s}^{-1}$ in Hanner et al. (1985) or $170 \mathrm{~kg} \mathrm{~s}^{-1}$ in Krishna Swamy (1991) near the maximum of activity. The higher dust production rates in this work may be partly caused by the large value for the maximum grain size compared to other publications. Krishna Swamy (1991) assumes a maximum grain size of only $100 \mu \mathrm{m}$. The mean dust to water mass ratio derived from all data given by LOCD is 4.8 . With the parameters used here, $67 \mathrm{P} / \mathrm{C}-\mathrm{G}$ is a very dusty comet.

Future analysis of the neck-line structure may lead to refined parameters of the dust particle properties. Large deviations from the usual parameters will lead to significant changes of the dust mass production rate. Furthermore, a large uncertainty comes from the assumption on the active surface fraction. If this fraction is larger than assumed, the maximum particle size that can be lifted from the nucleus reduces significantly and, therefore, the dust mass production rate. Varying the active fraction by $50 \%$ changes the dust mass production rates by nearly a factor of two. In case of very high dust mass loading of 


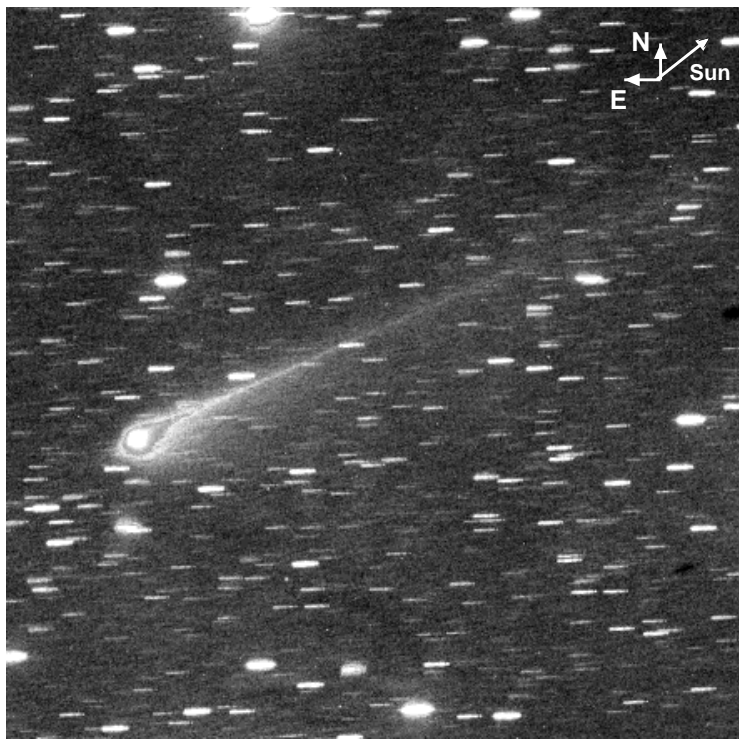

Fig. 3. $R$-filter image of comet 67P/C-G, obtained on March 27, 2003 (see Table 3). The field of view is $16.8^{\prime} \times 16.8^{\prime}, 36$ min exposure time.

the gas flow it could also be important to take the influence of the dust onto the gas flow (e.g. heat transfer) into account which was not done in our test particle approach. For these reasons, the results of our dust production rates have to be regarded as crude estimates since the dust properties of comet $67 \mathrm{P} / \mathrm{C}-\mathrm{G}$ are only poorly known up to now.

\section{Dust Coma morphology}

In Fig. 3, comet 67P/C-G is shown on March 27, 2003. An asymmetric coma and an extended neck-line structure can be seen since the Earth is very close to the comet's orbital plane $\left(2.9^{\circ}\right.$ south of the orbital plane in Fig. 3). The neckline can be detected over a length of approx. 10' corresponding to $7.4 \times 10^{5} \mathrm{~km}$ projected nucleocentric distance.

In order to reveal structures in the dust coma of comet $67 \mathrm{P} / \mathrm{C}-\mathrm{G}$, a mean radial intensity profile was subtracted from the comet images. To obtain the mean coma intensity profile, the comet image was transformed into polar coordinates with the optocenter as the center of the polar coordinate system. The mean profile from all position angles was fitted and subtracted. In Fig. 4, the mean intensity profile of the coma is shown for the observation on March 27, 2003, together with a fit to the profile. The mean profile varies not according to $i^{-1}$ where $i$ is distance from the optocenter in pixels, as given by the dashed line. A dependency according to $i^{-1}$ would be expected in case of isotropic emission of dust from the nucleus of a comet.

In Fig. 5, the deviation of the cometary coma intensity from the fitted mean intensity distribution is shown for the observations on March 7, 27, 28 and 30. The profiles shown are averaged in radial direction from 4 to 8 pixels from the optocenter on March 7 and from 5 to 9 pixels for all other dates to cover a similar range of projected nucleocentric distances. This corresponds to projected nucleocentric distances from $6655 \mathrm{~km}$ to $13314 \mathrm{~km}$, slightly varying for the different dates because

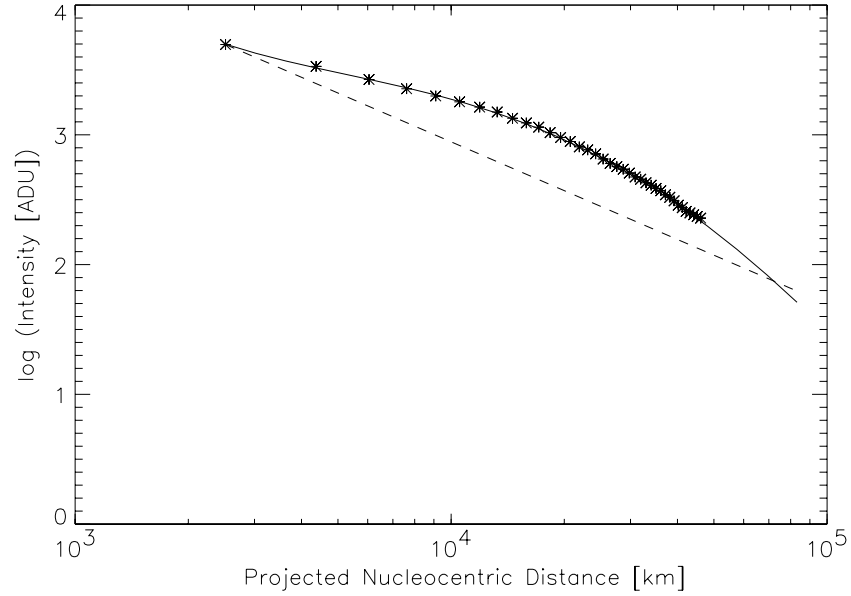

Fig. 4. Mean radial intensity profile for the innermost pixels of the coma of comet 67P/C-G on March 27, 2003. The solid line is a fit to the profile as used for coma subtraction. The dashed line gives a dependency according to $i^{-1}$, where $i$ is the pixel number counted from the optocenter (for optocenter, $i=0$ ).

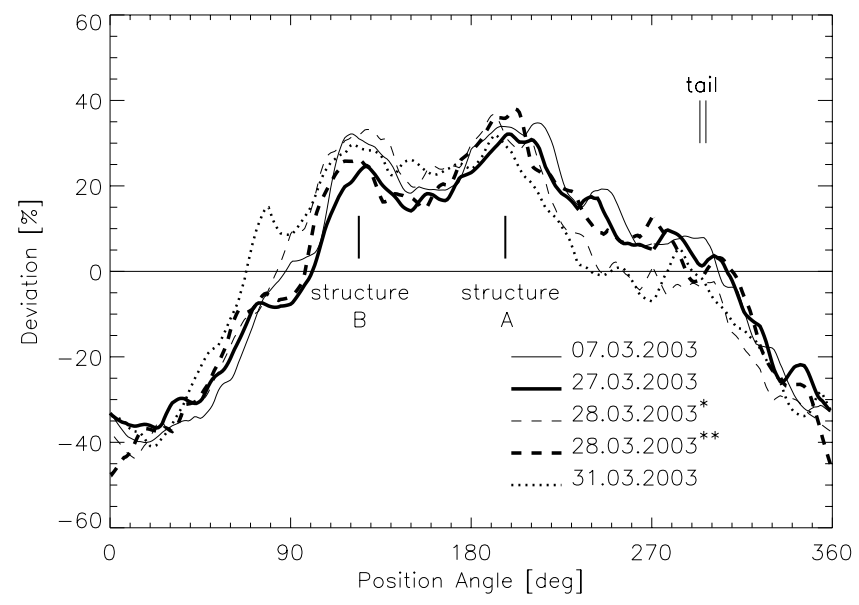

Fig. 5. Deviation from the mean coma intensity profile for observations in March, 2003. The azimuthal profiles shown are averaged between about $6657 \mathrm{~km}$ and $13314 \mathrm{~km}$ projected nucleocentric distance. The feature at $75^{\circ}$ on March 31 is a star trace. The position angle is measured from North towards East, the tail position varies between the two lines during the observation period. ${ }^{*}$ is the observation made in the morning, ${ }^{* *}$ made in the evening of March 28, 2003.

of the changing geocentric distance between the times of observation. The position angle is measured from North towards East.

In Fig. 5, an overall variation due to the coma asymmetry is obvious. The asymmetry leads to lower intensities compared to the mean profile at small and high position angles and higher intensities at intermediate angles.

Two weak features of increased intensity are visible in the coma marked as structure A and structure B in Fig. 5. These two structures appear in all of our observations at approximately the same position angles. For this reason, in addition to star traces having a different spatial direction, faint background stars can be ruled out as cause of these structures. An increased intensity in the coma at the position of structure A and B can also be detected in single 2-min exposures. Thus, it is unlikely 

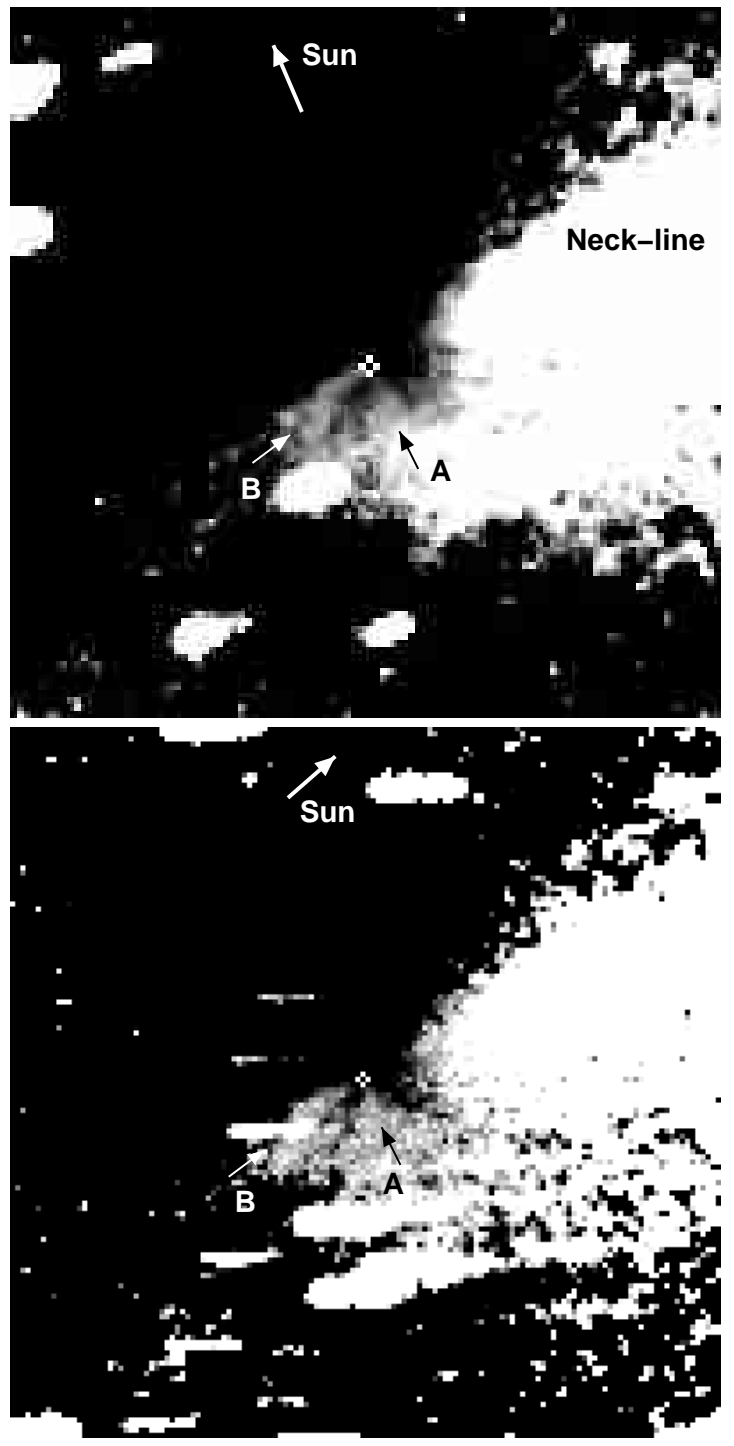

Fig. 6. Coma of comet 67P/C-G on March 7, 2003 (upper image) and on March 28, 2003, evening (lower image) after subtraction of the mean coma intensity. The structures A and B (see Fig. 5) are marked. A non-linear grayscale between $10 \%$ and $60 \%$ deviation from the mean intensity profile is applied. North is up, East to the left. The projected direction of the Sun is marked. The images cover $2.6^{\prime} \times 2.6^{\prime}$ corresponding to $1.66 \times 10^{5} \mathrm{~km}$ (upper image) and $1.91 \times 10^{5} \mathrm{~km}$ (lower image) projected width, respectively. The optocenter of the coma is located in the center.

that the structures are artefacts from image processing, e.g. resulting from shifting and co-addition of the images.

As an example, in Fig. 6 the result after subtraction of the mean coma intensity is shown for the observations made on March 7 and in the evening of March 28. Structure A and B are marked by arrows.

The presence of coma structures could indicate the presence of surface areas with enhanced activity. The low active surface fraction was regarded as an indication for localized active areas on the nucleus surface of $67 \mathrm{P} / \mathrm{C}-\mathrm{G}$ before (Lamy et al. 2003). However, since structure $B$ is oriented $180^{\circ}$ with respect to the extended neck-line, this structure could also be a part of the tail structure of comet $67 \mathrm{P} / \mathrm{C}-\mathrm{G}$.

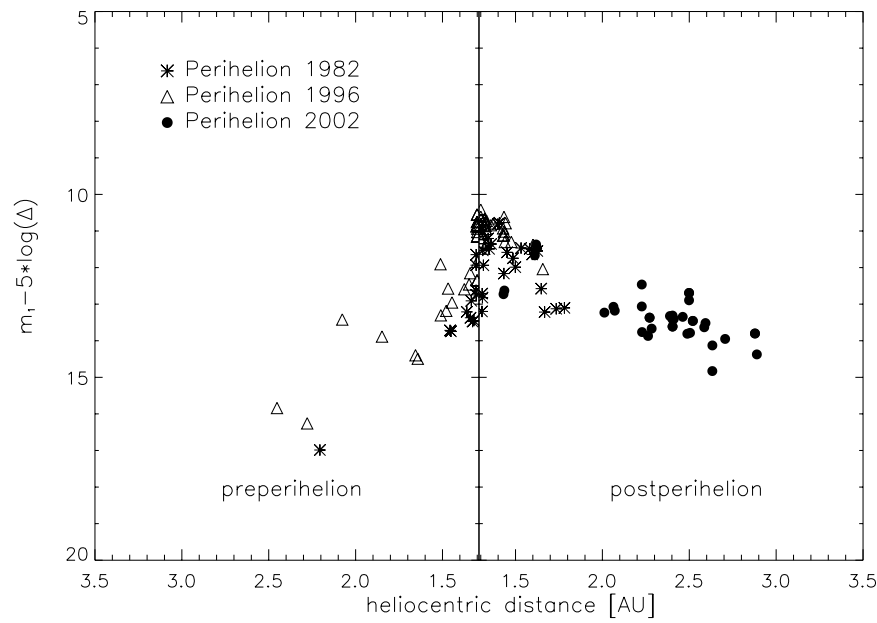

Fig. 7. Estimated magnitudes, $m_{1}$, of comet $67 \mathrm{P} / \mathrm{C}-\mathrm{G}$ for three perihelion passages. The magnitudes were taken from the ICQ/IAUC, the German and Italian Comet section archive and are corrected for the changing geocentric distances, $\Delta$.

\section{Summary and discussion}

The $\mathrm{CN}$ production rate and the $\operatorname{Af} \rho$ parameter derived from longslit spectra obtained 1996 are similar to values determined during the 1982 perihelion passage at similar heliocentric distances. Therefore, the comet's activity does not seem to vary strongly between recent apparitions.

Comet 67P/C-C showed a strong perihelion asymmetry. The enhanced activity postperihelion can be seen in the $\mathrm{CN}$ production rates and $\operatorname{Af} \rho$ values (see Fig. 2). The range of heliocentric distances covered by production rates for other species is small, but the asymmetry in activity seems to be present also for $\mathrm{OH}, \mathrm{C}_{2}, \mathrm{C}_{3}$ and $\mathrm{NH}$ in the data by LOCD and Cochran et al. (1992).

The lightcurve derived from optical observations shows the same development of activity with heliocentric distance for the 1982 and the 1996 apparition. In Fig. 7, visual lightcurves of the 1982, 1996 and 2002 perihelion passages are shown. The symbols give magnitude estimates $\left(m_{1}\right)$ as published by ICQ and IAUC. Additional magnitude estimates from the German Comet Section (VdS Fachgruppe Kometen) and Italian Comet Section (Sezione Comete UAI) archives are included. The data are corrected for changing geocentric distance. A similar dependence of activity from geocentric distance can be seen for the 1982 and 1996 apparition with the maximum of activity postperihelion.

It was suggested previously that storage of heat in cometary nuclei can cause perihelion asymmetries in their activity. Nevertheless, a steep increase of activity as seen for 67P/C-G may not result from this effect. Since the increase of activity occurs at the same point of the orbit in the 1982 and 1996 apparition (see Fig. 7), it is unlikely that outbursts cause the higher activity observed. The strong perihelion asymmetry may result from an inclination of the rotation axis with respect to the orbital plane. If the rotation axis in inclined, the illumination of parts of the surface can vary strongly along the comet's orbit. The sudden steep increase of activity at nearly the same point 
along the orbit in the 1982 and 1996 perihelion passage could be caused by an active surface area. If an active area on the nucleus surface is exposed to the sunlight from one point of the comet's orbit onwards, the total activity of the comet can increase in a short time. This is also the case if another hemisphere of the nucleus, having a less eroded surface, is exposed to the Sun on one part of the orbit. This explanation implies that the rotation axis of the nucleus is not perpendicular to the orbital plane. If the asymmetry is caused by active surface areas, the appearance of jet-like structures in the dust coma is likely.

In $R$-filter images of comet $67 \mathrm{P} / \mathrm{C}-\mathrm{G}$, obtained in March and May 2003, two weak jet-like features could be clearly detected. No variation of these features with time was observed during our observation period.

For the nucleus of comet $67 \mathrm{P} / \mathrm{C}-\mathrm{G}$ a rotation period of $12.3 \mathrm{~h}$ was determined by Lamy et al. (2003). Since the dates and times of our observations correspond to different rotational phases, the same visual appearance is not caused by unfavorable sampling of our observations.

One of these structures (structure B) could be part of the neck-line structure in which case it is not expected to vary significantly within this time period. But since both structures observed show no variation, it could also be possible that both result from one active area on the surface being the edges of a cone resulting from an active spot on the rotating nucleus. A scenario like this was proposed before to explain similar structures in the coma of comet Hale-Bopp (Sekanina \& Boehnhardt 1999). This explanation allows to put constraints on the orientation of the rotation axis of the nucleus. Since the axis is expected to be centered in the cone, the observed jet structures suggest an inclination of the projected rotational axis of approximately $40^{\circ}$ with respect to the orbital plane. However, at this point the orientation of the rotation axis is speculative and further observations and proper modeling of the coma hydrodynamics are required to obtain better constraints.

\section{References}

A'Hearn, M. F., Millis, R. L., Schleicher, D. G., Osip, D. J., \& Birch, P. V. 1995, Icarus, 118, 223

A'Hearn, M. F., Schleicher, D. G., Millis, R. L., Feldman, P. D., \& Thompson, D. T. 1984, AJ, 89, 579

Cochran, A. L. 1985, AJ, 90, 2609

Cochran, A. L., Barker, E. S., Ramseyer, T. F., \& Storrs, A. D. 1992, Icarus, 98, 151

Divine, N. 1981, ESA-SP, 174

Fink, U., Combi, M. R., \& Disanti, M. A. 1991, ApJ, 383, 356

Hanner, M. S., Tedesco, E., Tokunaga, A. T., et al. 1985, Icarus, 64, 11

Jewitt, D., \& Meech, K. J. 1986, AJ, 310, 937

Krishna Swamy, K. S. 1991, A\&A, 241, 260

Kurucz, R. L., Furenlid, I., Brault, J., \& Testerman, L. 1984, Solar Flux Atlas from 296 to $1300 \mathrm{~nm}$ (Tech. rept. National Solar Observatory, Sunspot, New Mexico)

Lamy, P. L., Toth, I., Weaver, H., Jorda, L., \& Kaasalainen, M. 2003, AAS/Division for Planetary Sciences Meeting, 35

Lemaitre, G., Kohler, D., Lacroix, D., Meunier, J. P., \& Vin, A. 1990, A\&A, 228, 546

Newburn, R. L., \& Spinrad, H. 1985, AJ, 90, 2591

Osip, D. J., Schleicher, D. G., \& Millis, R. L. 1992, Icarus, 98, 115

Rauer, H., Helbert, J., Arpigny, C., et al. 2003, A\&A, 397, 1109

Schleicher, D. G. 1983, Ph.D. Thesis

Sekanina, Z., \& Boehnhardt, H. 1999, Earth Moon and Planets, 78, 313

Storrs, A. D., Cochran, A. L., \& Barker, E. S. 1992, Icarus, 98, 163

Weiler, M., Rauer, H., Knollenberg, J., Jorda, L., \& Helbert, J. 2003 , A\&A, 403, 313 